\title{
Orbital-resolved spin model for thermal magnetization switching in rare-earth-based ferrimagnets
}

\author{
S. Wienholdt, ${ }^{1, *}$ D. Hinzke, ${ }^{1}$ K. Carva,,${ }^{2,3, \dagger}$ P. M. Oppeneer, ${ }^{2}$ and U. Nowak ${ }^{1, \ddagger}$ \\ ${ }^{1}$ Fachbereich Physik, Universität Konstanz, D-78457 Konstanz, Germany \\ ${ }^{2}$ Department of Physics and Astronomy, Uppsala University, Box 516, SE-751 20 Uppsala, Sweden \\ ${ }^{3}$ Charles University in Prague, Faculty of Mathematics and Physics, DCMP, Ke Karlovu 5, CZ-12116 Prague 2, Czech Republic
}

(Received 10 December 2012; revised manuscript received 10 June 2013; published 24 July 2013)

\begin{abstract}
The switching of rare-earth-based ferrimagnets triggered by thermal excitation is investigated on the basis of an atomistic spin model beyond the rigid-spin approximation, distinguishing magnetic moments due to electrons in $d$ and $f$ orbitals of the rare earth. It is shown that after excitation of the conduction electrons a transient ferromagneticlike state follows from a dissipationless spin dynamics where energy and angular momentum are distributed between the two sublattices. The final relaxation can then lead to a new state with the magnetization switched with respect to the initial state. The time scale of the switching event is to a large extent determined by the exchange interaction between the two sublattices.
\end{abstract}

DOI: 10.1103/PhysRevB.88.020406

PACS number(s): 75.10.Hk, 75.40.Mg, 75.50.Gg, 75.78.Jp

The quest for ever increasing speed of data procession has its bottleneck in data storage with current hard disk writing events being on the time scale of nanoseconds. Much quicker writing schemes have been demonstrated based on all-optical magnetization reversal mechanisms using circularly polarized laser light, with the helicity of the light determining the direction of magnetization in the written area. ${ }^{1-5}$ Most surprisingly, it has been demonstrated that even linearly polarized light can trigger a thermally driven switching in ferrimagnetic GdFeCo compounds ${ }^{6,7}$ via a so-called "ferromagneticlike state," where the rare-earth (RE) and transition-metal (TM) sublattice magnetizations are aligned parallel on a picosecond time scale.

With these experiments the theoretical understanding of magnetization dynamics in terms of the macroscopic LandauLifshitz-Gilbert (LLG) equation of motion has reached its limits. The short time scale of the laser pulse in connection with the high electron temperatures following the excitation lead to nonequilibrium processes where longitudinal magnetization dynamics becomes pronounced. ${ }^{2,8-13}$ A full theoretical explanation of the thermally driven switching process in ferrimagnets and, particularly of the transient ferromagneticlike state, is still missing, though first attempts of a description of longitudinal magnetization dynamics in two-sublattice systems have been proposed recently. ${ }^{10,14}$

In this Rapid Communication, we present a microscopic model for switching in RE-based ferrimagnets resting on basic principles as, e.g., the maximization of entropy and conservation of angular momentum in connection with an atomic spin model beyond the usual rigid-spin approximation. This model provides an improved understanding for the processes leading to the thermal switching in RE-based ferrimagnets. In particular, our atomistic spin-dynamics simulations reveal the importance of a two-spin treatment for the $\mathrm{Gd}$ atoms, having two distinct spins stemming from the $5 d$ and $4 f$ orbitals, and explain the origin of the ferromagneticlike state. The general idea to parametrize the magnetic exchange energy using an orbital-resolved spin model has been formulated earlier ${ }^{15,16}$ but here we apply it in actual spin model calculations.

For a first qualitative understanding we split the whole dynamic process according to the different times scales involved. (i) On the time scale of the laser pulse electronic processes govern the dynamics and lead to ultrafast demagnetization of the Fe sublattice typical for transition metals. ${ }^{17,18}$ The quantum mechanical processes underlying ultrafast demagnetization after femtosecond laser excitation are still up for debate. Several contributions are supposed to play a crucial role, e.g., Elliott-Yafet-like electron-phonon scattering, ${ }^{11,19}$ electronelectron spin flip scattering, ${ }^{20}$ as well as superdiffusive spin transport. $^{21}$ All these processes are a consequence of the "hot electrons" after the excitation. Here, it is important to note that the $4 f$ electrons of Gd, which carry most of the magnetic moment of the Gd sublattice, lie deep below the Fermi energy $y^{22,23}$ and cannot be directly excited with the laser energies used in Refs. 6 and 7. After the initial excitation, the degree of thermal excitation of the Fe sublattice must hence be much larger than the thermal disorder associated with the Gd degrees of freedom. In Gd the $5 d$ electrons can be excited, but those carry only a very small magnetic moment $\left(\approx 0.6 \mu_{\mathrm{B}}\right)$. However, the energy from thermal excitation of $5 d$ electrons can be transferred to the $4 f$ electrons via an intra-atomic exchange.

(ii) After that initial excitation the system of spin degrees of freedom is in a strong nonequilibrium state, where the Fe spins are much more thermally excited than the $\mathrm{Gd} f$ spins. As a consequence of the principle of maximum entropy even a closed system, where angular momentum and energy are conserved, must now show a relaxation by transfer of energy and angular momentum from the Fe to the Gd sublattice. As we will show in the following, this dissipationless relaxation leads to a state with the two sublattices alignedthe transient ferromagneticlike state-on a picosecond time scale.

(iii) On larger time scales of some picoseconds, dissipative relaxation processes lead to a loss of energy and angular momentum in the spin system with a relaxation back to a ferrimagnetic state. The relaxed state can be reversed with respect to the initial one.

In the following, we model the mechanisms above with an improved, realistic spin model which distinguishes $d$ and $f$ electrons of the RE and their degree of thermal excitation. This orbital-resolved spin model explains the experimental behavior with a switching process in the picosecond regime 


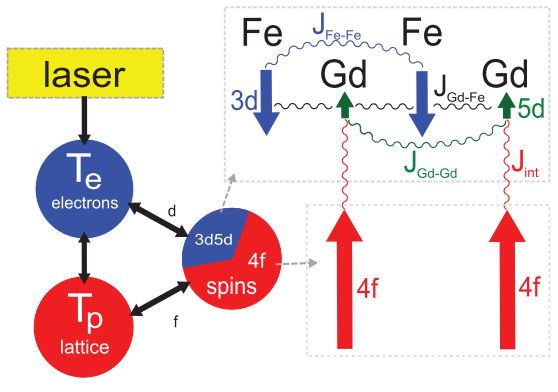

FIG. 1. (Color online) Sketches of the orbital-resolved spin model, distinguishing spins stemming from electrons in $5 d$ and $4 f$ orbitals of Gd (right-hand panel), and the coupling of the spin system to the electronic and phononic heat baths (left-hand panel).

and we will especially discuss the role of the dissipationless dynamics of the two sublattices.

The energy of the system in our model can still be parametrized in the form of scalar products between classical spins (like a Heisenberg exchange) but we introduce also an intra-atomic exchange between magnetic moments of the electrons in different orbitals. ${ }^{16}$ The Hamiltonian of the spin system is then expressed in the classical limit via unit vectors $\mathbf{S}_{i}$ and $\mathbf{S}_{i}^{\prime}$, where each represents the normalized magnetic moment stemming from the electrons in an orbital of the $i$ th atom. These are called (classical) spins in the following. We distinguish three types of spins (see Fig. 1): (i) the spins of the $3 d$ orbitals of the TM ( $3 d$ spins), (ii) the spins of $5 d$ orbitals ( $5 d$ spins), and (iii) of the $4 f$ orbitals of the RE ( $4 f$ spins). The $\mathbf{S}_{i}$ represent the $d$ spins (with magnetic moment $\mu_{\mathrm{s}}^{i}$ ) and the $\mathbf{S}_{i}^{\prime}$ represent the $4 f$ spins of Gd (with magnetic moment $\mu_{\mathrm{s}}^{\prime i}$ ). The model hence allows for longitudinal fluctuations on an atomic level going beyond the usual rigid-spin approximation. The Hamiltonian reads

$$
\mathcal{H}=-\sum_{\langle i j\rangle} \frac{J_{i j}}{2} \mathbf{S}_{i} \cdot \mathbf{S}_{j}-\sum_{i \in \mathrm{Gd}} J_{\mathrm{int}} \mathbf{S}_{i} \cdot \mathbf{S}_{i}^{\prime}-d_{z} \sum_{i}\left(S_{i}^{z}\right)^{2} .
$$

The first term represents the Heisenberg exchange between the $d$ spins of the different sublattices taking into account nearest neighbors (NN) and next nearest neighbors (NNN). The second term describes the exchange between the $5 d$ spins and the $4 f$ spins in the RE. Figure 1 shows the different couplings in our model. Note that the $4 f$ spins of the REdue to their high degree of localization-are only coupled via this intra-atomic exchange, which we calculated with $a b$ initio calculations and turns out to be about $J_{\text {int }}=130 \mathrm{meV}$. The $a b$ initio calculations have been performed using density functional theory in the local-spin density approximation, by comparing total energies computed for two constrained spin configurations having parallel or antiparallel $5 d$ and $4 f$ spin moments on $\mathrm{Gd}$. The calculations were carried out both for elemental hcp Gd as well as for the prototypical cubic Laves phase $\mathrm{GdFe}_{2}$. The intra-atomic exchange $J_{\text {int }}$ was found to be very similar for $\mathrm{Gd}$ and $\mathrm{GdFe}_{2}$.

In our simulations the $d$ spins are arranged on a cubic (C15) Laves phase, reminiscent of $\mathrm{GdFe}_{2}$, where the number of TM atoms is twice the number of RE atoms. Each TM atom has six TM atoms as NN and six RE atoms as NNN, while each RE atom has 12 TM atoms as NN and four RE atoms as NNN. The exchange constants between the $d$ spins of different atoms are set to $J_{\mathrm{TM}-\mathrm{TM}}=32.5 \mathrm{meV}, J_{\mathrm{RE}-\mathrm{RE}}=7.8 \mathrm{meV}$, and $J_{\mathrm{RE}-\mathrm{TM}}=$ $-3.25 \mathrm{meV}$. The atomistic magnetic moments are set to $\mu_{\mathrm{s}}^{\mathrm{Fe}}=$ $1.92 \mu_{\mathrm{B}}$ and $\mu_{\mathrm{s}}^{\mathrm{Gd}}=0.63 \mu_{\mathrm{B}}$ for the $d$ spins and $\mu_{\mathrm{s}}^{\prime \mathrm{Gd}}=7 \mu_{\mathrm{B}}$ for the $4 f$ spins of Gd. The third term in Eq. (1) represents a uniaxial anisotropy with anisotropy constant $d_{z}=1.3 \mathrm{meV}$. These constants result in a Curie temperature $T_{\mathrm{C}}=560 \mathrm{~K}$ and a magnetization compensation temperature $T_{\mathrm{M}}=300 \mathrm{~K}$. These values are representative of a GdFeCo alloy. ${ }^{6}$ As the percentage of Co is small ( $\sim 9 \%)$, only $\mathrm{Gd}$ and Fe sublattices are taken into account.

We consider Langevin dynamics, i.e., we numerically solve the stochastic LLG equation of motion for each spin,

$$
\begin{aligned}
\dot{\mathbf{S}}_{i}= & -\frac{\gamma_{i}}{\left(1+\alpha_{i}^{2}\right) \mu_{\mathrm{s}}^{i}} \mathbf{S}_{i} \times \mathbf{H}_{i}(t) \\
& -\frac{\alpha_{i} \gamma_{i}}{\left(1+\alpha_{i}^{2}\right) \mu_{\mathrm{s}}^{i}} \mathbf{S}_{i} \times\left[\mathbf{S}_{i} \times \mathbf{H}_{i}(t)\right] .
\end{aligned}
$$

Here, $\gamma_{i}$ denotes the gyromagnetic ratio associated with the spin at site $i$. For the $4 f$ spins of Gd we have the equivalent equation with the primed quantities. Thermal fluctuations are included via an additional white-noise term ${ }^{24}$ so that $\mathbf{H}_{i}(t)=$ $-\frac{\partial \mathcal{H}}{\partial \mathbf{S}_{i}}+\zeta_{i}(t)$ is the effective field and the thermal noise term $\zeta_{i}$ fulfills

$$
\left\langle\zeta_{i}(t)\right\rangle=0, \quad\left\langle\zeta_{i \eta}(0) \zeta_{j \theta}(t)\right\rangle=\delta_{i j} \delta_{\eta \theta} \delta(t) 2 \alpha_{i} k_{\mathrm{B}} T_{i} \mu_{\mathrm{s}}^{i} / \gamma_{i} .
$$

The excitation triggered by the laser pulse is simulated as follows: the laser pulse excites the electronic system of Fe and Gd and so leads to a nonequilibrium between the temperatures of the electron gas, $T_{\mathrm{e}}$, and the temperature of the lattice, $T_{\mathrm{p}}$. The evolution of these temperatures is calculated via the well-known "two temperature model" $(2 \mathrm{TM}),{ }^{25,26}$ which shows good agreement with the measured electron and lattice dynamics in transition metals ${ }^{27}$ as well as in Gd. ${ }^{28}$ Here, we use the 2TM as already described and applied in Ref. 4. The different heat baths are then coupled via a phenomenological damping constant $\alpha_{i}$ to the spin system [see Eq. (3)]. All the microscopic demagnetization processes can be approximately included in the phenomenological damping constant $\alpha_{i}$. This approach has been shown to describe ultrafast demagnetization in good agreement with experiments either in atomistic LLG simulations ${ }^{18}$ or with simulations via the Landau-LifshitzBloch equation. ${ }^{29}$ The latter are similar to the "microscopic three temperature model" introduced recently by Koopmans et al. ${ }^{11,12}$ However, the energetically low-lying $f$ states of the $\mathrm{Gd}$, at about $8 \mathrm{eV}$ binding energy, ${ }^{22,23}$ cannot be excited by the laser energies used in Refs. 6 and 7. We take this into account by coupling the Gd $4 f$ spins only to the phononic heat bath, and only the $d$ spins of both Fe and Gd to the electronic heat bath (see again Fig. 1 and Ref. 30 for a similar discussion). Nevertheless, we use the same damping constants $\alpha_{i}=\alpha_{i}^{\prime}=0.02$ for simplicity. Note that this value leads to an effective damping parameter $\alpha_{\text {eff }}=0.06$ for the whole ferrimagnet, which is a reasonable value for $\mathrm{GdFeCo}$ compounds. ${ }^{31,32}$ Since during the initial excitation the electron temperature is much higher than the phonon temperature the magnetic moments of the Fe are much more excited than those of the Gd, especially since most of the magnetic moment of the $\mathrm{Gd}(92 \%)$ comes from the $f$ electrons. 


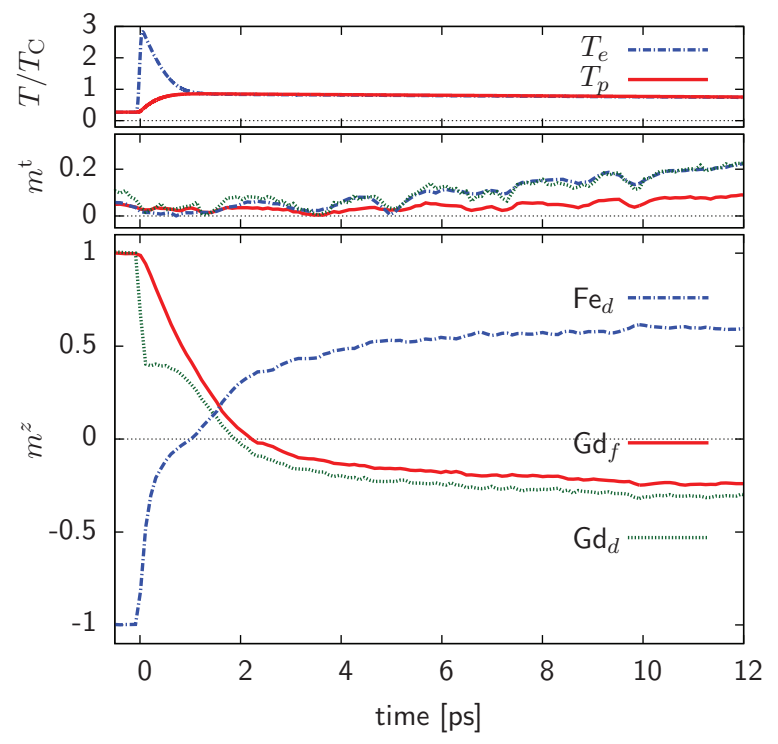

FIG. 2. (Color online) Thermally driven switching of the sublattice magnetization in $\mathrm{GdFe}$, using a spin model of 81000 atoms distinguishing magnetization stemming from $5 d$ and $4 f$ orbitals of Gd and $3 d$ orbitals of Fe. Electron $\left(T_{\mathrm{e}}\right)$ and phonon $\left(T_{\mathrm{p}}\right)$ temperatures are shown as well as the transverse $\left(\mathrm{m}^{t}\right)$ and longitudinal $\left(\mathrm{m}^{z}\right)$ magnetization dynamics of the different sublattices and orbitals.

The dynamics of a spin system of 81000 atoms $(15 \times$ $15 \times 15$ unit cells) with periodic boundary conditions, which follows from a thermal excitation, is presented in Fig. 2. The upper part shows the electron and phonon temperature with a maximum electron temperature of $1585 \mathrm{~K}$. Regarding the dynamics of the spin system it is first of all remarkable that the magnetization of the Fe sublattice decays much quicker than the magnetization of the Gd $4 f$ electrons. This is in agreement with recent measurements. ${ }^{6}$ Note that we have normalized the magnetization to unity at time $t=0$, but since the magnetic moment of Gd comes mostly from $4 f$ electrons, their signal is most of all relevant and only that contribution is usually measured with element-specific methods. ${ }^{6}$ The reason for this larger time scale is on the one hand the larger magnetic moment that goes into the noise term [see Eq. (3)] but also the fact that the $4 f$ spins are not coupled to the electronic heat bath with its higher peak electron temperatures.

Due to the different demagnetization times of the different sublattices, this initial demagnetization ends in a strongly nonequilibrium state after $1 \mathrm{ps}$. On that time scale, electron and phonon temperatures are nearly equilibrated and have fallen below $T_{\mathrm{C}}$ again. The spins of the Fe sublattice are completely demagnetized, which implies that they will start to order again, whereas the spins of the Gd sublattice are still rather ordered and continue their demagnetization. The remagnetization dynamics of $\mathrm{Fe}$ taking place subsequently leads to a state where the Gd spins and the Fe spins are aligned - a transient ferromagneticlike state. The occurrence of this transient state is a direct consequence of angular momentum conservation in the spin system. Of course, the LLG equation of motion that we use in our simulations does not strictly conserve angular momentum due to dissipative processes which are described phenomenologically via the damping term. However, since this dissipative term is usually small, with damping parameters of the order of $10^{-2} \cdots 10^{-5}$ the time scale for dissipation is longer, and on shorter times scales nondissipative processes dominate.

To investigate this further, we also performed nondissipative simulations with zero damping in a simplified model with only two sublattices. Still highly nontrivial dynamics is found to exist for ferrimagnets, which is shown in Fig. 3. Here, we simulate a ferrimagnetic model of 64000 spins with zero damping and initial conditions where one sublattice is completely ordered while the other one is perfectly disordered-a random spin configuration. In this model the spins are arranged on a simple cubic lattice. We set the magnetic moments to $\mu_{\mathrm{s}}^{\mathrm{RE}}=2 \mu_{\mathrm{s}}^{\mathrm{TM}}=2 \mu_{\mathrm{B}}$ and the exchange constants to $J_{\mathrm{TM}-\mathrm{TM}}=3 J_{\mathrm{RE}-\mathrm{RE}}=-3 J_{\mathrm{RE}-\mathrm{TM}}=7 \mathrm{meV}$. This serves as toy model for a ferrimagnetic FeGd sample directly after the initial excitation where the Fe spins are rather random while the Gd spins are still rather ordered due to their slower dynamics. The dynamics of our model starts consequently at zero magnetization for the Fe sublattice and with a finite value for the Gd sublattice. The fact that the total angular momentum is conserved in this toy model (also shown in Fig. 3) in connection with the exchange of energy (shown in the upper part of Fig. 3) leads necessarily to a ferromagneticlike state, since the change of magnetization of the Gd (negative) must be balanced by a corresponding positive change of the $\mathrm{Fe}$ magnetization. The redistribution of the energy is a direct consequence of the maximization of entropy. The dynamics is driven by the exchange coupling between TM and RE moments via the precession of atomic moments, which on average have almost no transverse moment. This takes place on time scales less than one ps, which means much faster than the usual dissipative processes. A similar behavior-a dissipationless exchange of energy and angular momentum between the sublattices-is qualitatively described by the so-called Baryakhtar equation ${ }^{10,33}$ but not by the recently derived two-sublattice Landau-Lifshitz-Bloch equation, which
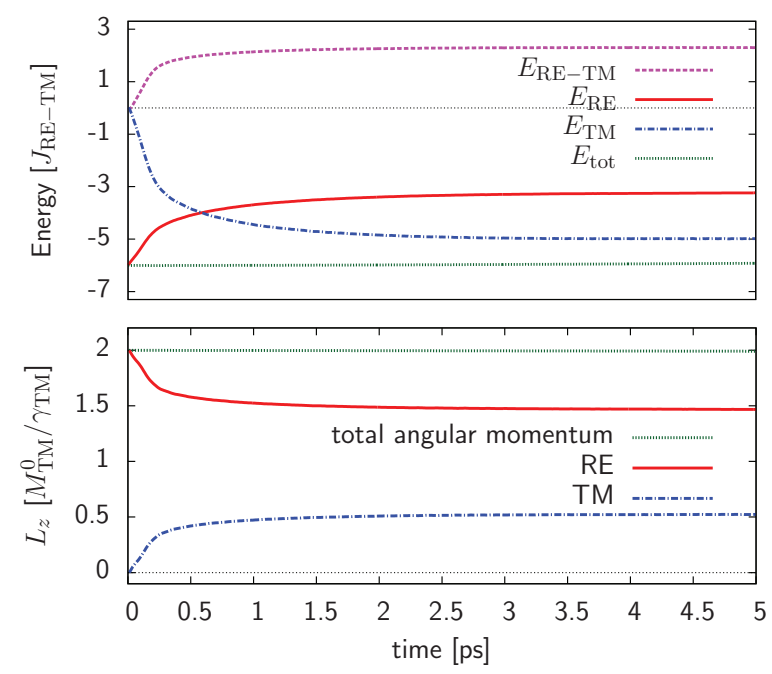

FIG. 3. (Color online) Dissipationless spin dynamics of two sublattices of a generic ferrimagnet: transfer of energy (top panel) and angular momentum (bottom panel) occur, keeping the total angular momentum and the total energy constant. The dynamics leads to a ferromagneticlike state. 


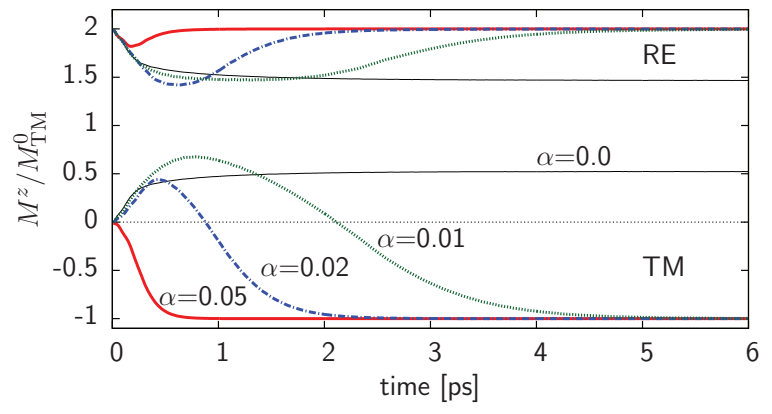

FIG. 4. (Color online) Nonequilibrium spin dynamics of the two sublattices of a ferrimagnet for different values of $\alpha$ : for small values of $\alpha$ the dissipationless dynamics in the first few hundred femtoseconds leads to a transient ferromagneticlike state. For $\alpha \geqslant$ 0.05 , this phenomenon does not occur.

describes no longitudinal dynamics at zero damping. ${ }^{14}$ We assume that the latter equation does not apply to nonequilibrium situations in which the energy and angular momentum transfer between the sublattices would dominate the dynamics.

To investigate the contribution of the dissipationless redistribution of energy and angular momentum in a model including dissipation effects, we repeated the zero-temperature simulations described above, with one sublattice completely demagnetized and the other completely ordered as initial conditions, for different values of the damping constant $\alpha$. Figure 4 shows that in the first few hundred femtoseconds the dynamics for lower damping constants follows the dissipationless dynamics with $\alpha=0.0$ leading to a transient ferromagneticlike state. Only for higher values $\alpha \geqslant 0.05$ the damping term becomes dominant and this phenomenon does not occur. The dynamics leads then directly into the ferrimagnetic ground state. This clearly shows that even in a system with relatively high damping constants $(\alpha=0.02)$ a strong nonequilibrium state via dissipationless dynamics relaxes first towards a transient ferromagneticlike state on a time scale of less than one picosecond before it relaxes finally into the ferrimagnetic state.

Coming back to the more realistic orbital-resolved spin model simulations shown in Fig. 2 we note the small kink in the curve of the Fe sublattice magnetization, exactly where the electron temperature goes below the Curie temperature. At this point the Fe sublattice starts to remagnetize, and, as described above, this happens on a ps time scale via dissipationless dynamics. Therefore, a ferromagneticlike state necessarily results. The magnetization of the Gd sublattice is still above its equilibrium value for this temperature, so that the dissipation processes support the dissipationless dynamics, which in combination finally lead to an almost complete demagnetization of the Gd sublattice. This means that the mechanisms leading to the switching are demagnetization-remagnetization processes, which, for the sublattice magnetization as a whole, have been shown to evolve linearly. ${ }^{18}$ Figure 2 shows the transverse magnetization $\left(\mathrm{m}^{t}\right)$ of the different sublattices, which are not exactly zero due to thermal fluctuations and finite size effects, but remain very small during switching. Once the system reaches this state the further dynamics will be governed by dissipation processes with the system relaxing slowly back to a ferrimagnetic state with switched sublattices.

To conclude, the thermally driven spin switching of REbased ferrimagnets can be well described on the basis of a physically realistic orbital-resolved spin model, distinguishing electrons in $d$ and $f$ orbitals of the RE. We have shown that a distinct treatment of the spins of different orbitals contributes significantly to the different demagnetization times of the strongly coupled $\mathrm{Fe}$ and $\mathrm{Gd}$ sublattice. As a consequence our simulations show the existence of a large transient ferromagneticlike state of the same magnitude seen in the experiments but not in former simulations. ${ }^{6,7}$ This means in particular that the exchange coupling between $4 f$ and $5 d$ spins in $\mathrm{Gd}$ is sufficient to revert the large magnetic moment of the Gd $4 f$ shell inaccessible to the pump laser on a picosecond time scale. The pronounced thermal excitation of $d$ electrons drives the system into a nonequilibrium state which by a dissipationless spin dynamics, where energy and angular momentum are distributed between the sublattices, relaxes into a transient ferromagneticlike state on time scales of just a picosecond. The short time scale of this process is explained by the fact that the redistribution of angular momentum is driven by the exchange interaction between the sublattices, without any need for much slower dissipation processes. The final dissipative relaxation then leads into a new state with the sublattice magnetizations switched with respect to the initial state. The switching of the sublattices leads finally also to a reversed magnetization when the system is cooled down to the initial temperature which it had prior to being hit by the laser pulse.

This work was supported by the European Commission under Contract No. 281043 "FemtoSpin." The authors in Konstanz acknowledge also funding by the Center for Applied Photonics at the University of Konstanz. The authors at Uppsala acknowledge support through the Swedish National Infrastructure for Computing (SNIC).

\footnotetext{
*soenke.wienholdt@uni-konstanz.de

${ }^{\dagger}$ karel.carva@fysik.uu.se

${ }^{\ddagger}$ ulrich.nowak@uni-konstanz.de

${ }^{1}$ C. D. Stanciu, F. Hansteen, A. V. Kimel, A. Kirilyuk, A. Tsukamoto,

A. Itoh, and Th. Rasing, Phys. Rev. Lett. 99, 047601 (2007).

${ }^{2}$ K. Vahaplar, A. M. Kalashnikova, A. V. Kimel, D. Hinzke,

U. Nowak, R. Chantrell, A. Tsukamoto, A. Itoh, A. Kirilyuk, and

Th. Rasing, Phys. Rev. Lett. 103, 117201 (2009).
}

${ }^{3}$ S. Alebrand, A. Hassdenteufel, D. Steil, M. Cinchetti, and M. Aeschlimann, Phys. Rev. B 85, 092401 (2012).

${ }^{4}$ K. Vahaplar, A. M. Kalashnikova, A. V. Kimel, S. Gerlach, D. Hinzke, U. Nowak, R. Chantrell, A. Tsukamoto, A. Itoh, A. Kirilyuk, and Th. Rasing, Phys. Rev. B 85, 104402 (2012).

${ }^{5}$ A. R. Khorsand, M. Savoini, A. Kirilyuk, A. V. Kimel, A. Tsukamoto, A. Itoh, and Th. Rasing, Phys. Rev. Lett. 108, 127205 (2012). 
${ }^{6}$ I. Radu, K. Vahaplar, C. Stamm, T. Kachel, N. Pontius, H. A. Dür, T. A. Ostler, J. Barker, R. F. L. Evans, R. W. Chantrell, A. Tsukamoto, A. Itoh, A. Kirilyuk, Th. Rasing and A. V. Kimel, Nature (London) 472, 205 (2011).

${ }^{7}$ T. A. Ostler et al., Nat. Commun. 3, 666 (2012).

${ }^{8}$ U. Atxitia, O. Chubykalo-Fesenko, N. Kazantseva, D. Hinzke, U. Nowak, and R. W. Chantrell, Appl. Phys. Lett. 91, 232507 (2007).

${ }^{9}$ N. Kazantseva, D. Hinzke, R. W. Chantrell, and U. Nowak, Europhys. Lett. 86, 27006 (2009).

${ }^{10}$ J. H. Mentink, J. Hellsvik, D. V. Afanasiev, B. A. Ivanov, A. Kirilyuk, A. V. Kimel, O. Eriksson, M. I. Katsnelson, and Th. Rasing, Phys. Rev. Lett. 108, 057202 (2012).

${ }^{11}$ B. Koopmans et al., Nat. Mater. 9, 259 (2010).

${ }^{12}$ U. Atxitia and O. Chubykalo-Fesenko, Phys. Rev. B 84, 144414 (2011).

${ }^{13}$ A. Mekonnen, A. R. Khorsand, M. Cormier, A. V. Kimel, A. Kirilyuk, A. Hrabec, L. Ranno, A. Tsukamoto, A. Itoh, and Th. Rasing, Phys. Rev. B 87, 180406(R) (2013).

${ }^{14}$ U. Atxitia, P. Nieves, and O. Chubykalo-Fesenko, Phys. Rev. B 86, 104414 (2012).

${ }^{15}$ V. P. Antropov, M. I. Katsnelson, B. N. Harmon, M. van Schilfgaarde, and D. Kusnezov, Phys. Rev. B 54, 1019 (1996).

${ }^{16}$ M. I. Katsnelson and A. I. Lichtenstein, Phys. Rev. B 61, 8906 (2000).

${ }^{17}$ E. Beaurepaire, J.-C. Merle, A. Daunois, and J. Y. Bigot, Phys. Rev. Lett. 76, 4250 (1996).

${ }^{18}$ N. Kazantseva, U. Nowak, R. W. Chantrell, J. Hohlfeld, and R. Rebei, Europhys. Lett. 81, 27004 (2008).

${ }^{19}$ D. Steiauf and M. Fähnle, Phys. Rev. B 79, 140401 (2009).
${ }^{20}$ M. Krauß, T. Roth, S. Alebrand, D. Steil, M. Cinchetti, M. Aeschlimann, and H. C. Schneider, Phys. Rev. B 80, 180407(R) (2009).

${ }^{21}$ M. Battiato, K. Carva, and P. M. Oppeneer, Phys. Rev. Lett. 105, 027203 (2010).

${ }^{22}$ M. Wietstruk, A. Melnikov, C. Stamm, T. Kachel, N. Pontius, M. Sultan, C. Gahl, M. Weinelt, H. A. Dürr, and U. Bovensiepen, Phys. Rev. Lett. 106, 127401 (2011).

${ }^{23}$ R. Carley, K. Döbrich, B. Frietsch, C. Gahl, M. Teichmann, O. Schwarzkopf, P. Wernet, and M. Weinelt, Phys. Rev. Lett. 109, 057401 (2012)

${ }^{24}$ U. Nowak, in Handbook of Magnetism and Advanced Magnetic Materials, Vol. 2, Micromagnetism, edited by H. Kronmüller and S. Parkin (John Wiley \& Sons Ltd., Chichester, 2007).

${ }^{25}$ M. I. Kaganov, I. M. Lifshitz, and L. V. Tanatarov, Sov. Phys. JETP 4, 173 (1957).

${ }^{26}$ M. Anisimov et al., Sov. Phys. JETP 39, 359 (1974).

${ }^{27}$ J. Hohlfeld, S.-S. Wellershoff, J. Güdde, U. Conrad, V. Jähnke, and E. Matthias, Chem. Phys. 251, 237 (2000).

${ }^{28}$ U. Bovensiepen, J. Phys.: Condens. Matter 19, 083201 (2007).

${ }^{29}$ U. Atxitia, O. Chubykalo-Fesenko, J. Walowski, A. Mann, and M. Münzenberg, Phys. Rev. B 81, 174401 (2010).

${ }^{30}$ M. Sultan, U. Atxitia, A. Melnikov, O. Chubykalo-Fesenko, and U. Bovensiepen, Phys. Rev. B 85, 184407 (2012).

${ }^{31}$ C. D. Stanciu, A. V. Kimel, F. Hansteen, A. Tsukamoto, A. Itoh, A. Kiriliyuk, and Th. Rasing, Phys. Rev. B 73, 220402(R) (2006).

${ }^{32}$ F. Schlickeiser, U. Atxitia, S. Wienholdt, D. Hinzke, O. ChubykaloFesenko, and U. Nowak, Phys. Rev. B 86, 214416 (2012).

${ }^{33}$ V. G. Baryakhtar, Zh. Eksp. Teor. Fiz. 94, 196 (1988) [Sov. Phys. JETP 67, 757 (1988)]. 